\title{
M 66224
}

\section{SIMULTANEOUS PARTICLE AND FIELD OBSERV ATIONS OF FIELD-ALIGNED CURRENTS}

FREDERICK W BERKO

ROBERT A HOFFMAN

RANDE K BURTON

ROBERT E. HOLZER

(NASA-TM-X-66224) SIMULTANEOUS PARTICLE

AND FIELD OBSERVATIONS OF FIELD-ALIGNED

CURRENTS (NASA) 34 P HC $\$ 3.75$ CSCL 08N 


\title{
SIMULTANEOUS PARTICLE AND \\ FIELD OBSERVATIONS OF \\ FIELD-ALIGNED CURRENTS
}

\author{
By \\ Frederick W. Berko \\ Robert A. Hoffman \\ Laboratory for Space Physics \\ NASA-Goddard Space Flight Center \\ Greenbelt, Md. 20771 \\ Rande $\mathrm{K}$. Burton \\ Robert E. Holzer \\ Institute of Geophysics and Planetary Physics \\ University of California \\ Los Angeles, California 90024
}

APRIL 1973

$I$ 


\section{ABSTRACT}

Simultaneous measurements of low-energy precipitating electrons and magnetic fluctuations from the low-altitude polar orbiting satellite 0GO-4 have been compared. Analys is of the two sets of experimenta1 data for isolated events led to the classification of high-latitude field-aligned currents as purely temporal or purely spatial variations. Magnetic field disturbances calculated using these simple current models and the measured particle fluxes were in good agreement with measured field values. While fluxes of greater than $1 \mathrm{keV}$ electrons are detected primarily on the nightside, magnetometer disturbances indicative of field-aligned currents were seen at al1 local times, both in the visual auroral regions and dayside polar cusp. Thus electrons with energies less than $\sim 1 \mathrm{keV}$ are the prime charge carriers in high-latitude dayside fieldaligned currents. The satellite measurements are in good agreement with previously measured field-aligned current values and with values predicted from several models involving magnetospheric field-aligned currents.

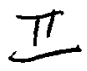




\section{INTRODUCTION}

Measurements of field-aligned fluxes of low-energy electrons at high latitudes have been made by several low-altitude satellite experiments. In addition to the direct particle measurements, such as those reported by Hoffman and Evans (1968) and Ackerson and Frank (1972), indirect evidence of field-aligned particle currents has been obtained from magnetometer observations. Transverse magnetic disturbances observed at $1100 \mathrm{~km}$ altitude in the auroral region by a satellite-borne magnetometer were reported by Zmuda et al. (1966). In later work (Zmuda et al., 1967, Armstrong and Zmuda, 1970), these high latitude disturbances were shown to be likely manifestations of the effects of field-aligned sheet currents.

Additional observations of field-aligned particle fluxes and of field-aligned currents have been reported by sounding rocket investigators. Among those detecting fluxes of precipitating particles peaked in the direction of the local magnetic field were Chase (1970), O'Brien and Reasoner (1971), Vondrak et a1. (1971), Whalen and McDiarmid (1972), and Evans et a1. (1972). Measurements indicating the existence of highlatitude field-aligned currents have been reported by Park and Cloutier (1971), and both field-aligned particle fluxes and field-aligned currents were detected by Choy et a1. (1971) and cloutier et a1. (1970).

A statistical study indicating the spatial extent of transverse magnetic disturbances (which Cummings and Dessler, 1967, explained to be due to currents flowing along magnetic field lines) has been performed by Zmuda et a1. (1970). The spatial distribution and spectral characteristics of $2.3 \mathrm{keV}$ field-aligned electrons have been presented in a statistical study by Berko (1973). 
In this paper, we will present simultaneous high time resolution measurements of precipitating particles and the magnetic effects of fieldaligned currents from two experiments aboard the polar-orbiting satellite 0G0-4. By comparing simultaneous particle detector and search coil magnetometer data, we will show that the 0G0-4 particle detectors usually measured the majority of the field-aligned currents in the high latitude region. The current magnitudes derived from the particle detectors compare favorably with published values of field-aligned currents, and are shown to have the correct magnitudes for the field-aligned portions of magnetospheric current system models.

\section{EXPERIMENT DESCRIPTIONS}

The 0G0-4 satellite was launched on 28 July 1967 into a nearly circular low-altitude polar orbit with an apogee of $\sim 908 \mathrm{~km}$ and a perigee of $412 \mathrm{~km}$. This orbit had an inclination of $86^{\circ}$, which, combined with the motion of the earth around the sun, caused a precession of the orbital plane from its initial dusk-dawn orientation through all local times at the rate of about $1 \frac{1}{2}^{\circ}$ per day. The satellite was body stabilized with the $t z$ axis pointing towards the earth and the sun in the $-y,-z$ quadrant. (Lower case letters will always refer to spacecraft axes.) The velocity vector of the spacecraft depended upon the local time of the orbit. In the noon-midnight meridian it was either in the ty or -y direction, whereas in a dawn-dusk meridian it was in a $+x$ or $-x$ direction. This is an important factor in the analysis of the search coil magnetometer data. Satellite telemetry was at either $4 \mathrm{kbps}$ (tape recorder playback or real time), or at 16 or $64 \mathrm{kbps}$ real time rates; only high time resolution 
data at the two latter real time telemetry rates were found to be suitable for this study.

The OGO-4 Auroral Particles Experiment (Hoffman and Evans, 1968) consisted of an array of 8 detectors, each comprised of an electrostatic analyzer for species and energy selection and a Bendix channel electron multiplier as the particle detector (Evans, 1965). Four of the detectors, (the "energy detectors"), were positioned to point radially away from the earth and measured either electrons or protons in narrow energy bands centered at $0.7,2.3,7.3$, and $23.8 \mathrm{keV}$. Three other detectors (the "angle detectors"), which measured charged particles at $2.3 \mathrm{keV}$ were positioned $30^{\circ}, 60^{\circ}$, and $90^{\circ}$ from the earth-spacecraft vector; the eighth detector served as a background detector.

Each of the "energy" and "angle" detectors had bandpasses of $-13 \%$ and $+19 \%$ about their center energy. At the high 1 atitudes where almost all of the data used for this paper were taken, the energy detectors were oriented approximately parallel to the local magnetic field, and primarily measured particles with pitch angles near $0^{\circ}$.

The 0G0-4 Search Coil Magnetometer experiment (Frandsen et al., 1969) consisted of a set of three sensors, the $X$ and $Y$ search coils oriented $45^{\circ}$ and $135^{\circ}$ respectively to the earth-satellite radius vector in the spacecraft $x, z$ plane with the $Z$ search coil oriented along the spacecraft ty direction, thus making an orthogonal set. (Upper case letters will always refer to search coil axes.) Response of the search coils was linear with frequency up to about $50 \mathrm{~Hz}$ at the highest telemetry rate, where the instrument Nyquist frequency was $83 \mathrm{~Hz}$. Above $50 \mathrm{~Hz}$, the response fell off at the rate of $12 \mathrm{db} /$ oct to prevent aliasing. Search coil magnetometer experiment data presented in this paper are from 
the waveform channel, in which the signal on each axis was sampled at intervals dependent on the satellite telemetry rate.

A search coil magnetometer measures an induced voltage given by:

$$
V=-\frac{d}{d t}(A \vec{r} \cdot \vec{B})
$$

where $A$ is the effective coil area and $\vec{n}$ is a unit vector along the coil axis. The measured quantity is thus

$$
\mathrm{V}=-\mathrm{A}\left\{\frac{\mathrm{d} \overrightarrow{\mathrm{n}}}{\mathrm{dt}} \cdot \overrightarrow{\mathbf{B}}+\overrightarrow{\mathrm{n}} \cdot\left[\frac{\partial \overrightarrow{\mathrm{B}}}{\partial t}+(\overrightarrow{\mathrm{v}} \cdot \vec{\nabla}) \overrightarrow{\mathrm{B}}\right]\right\},
$$

where the three terms are due to the rotation of the coil in a stationary field, the time variation of $\vec{B}$, and the translational motion with velocity $\vec{v}$ in a spatially varying field. For a non-rotating spacecraft, the first term vanishes, and thus

$$
\mathrm{V}=-\mathrm{A} \overrightarrow{\mathrm{n}} \cdot \overrightarrow{\mathrm{B}}
$$

where

$$
\overrightarrow{\dot{B}}=\frac{d \vec{B}}{d t}=\frac{\partial \vec{B}}{d t}+(\vec{v} \cdot \vec{\nabla}) \vec{B}
$$

In general, the temporal and spatial parts are not uniquely separable. Therefore, in our attempts at analyzing the magnetic fluctuations seen in the search coil data, we have assumed certain simple current mode1s: purely temporal variations, and cylindrical and sheet current spatial variations. Figure 1 illustrates these three model current variations, their resultant magnetic fields, and the derivative of $B$, which is related to the signal from the search coils through equation (3). 
Field-aligned currents at high latitudes where the magnetic field lines are nearly vertical would produce a magnetic field in the horizontal direction, and thus the $X$ and $Y$ search coil outputs would show fluctuations exactly in phase and in the same sense. The $Z$ axis sensor, oriented perpendicular to the plane containing the $\mathrm{X}$ and $\mathrm{Y}$ search coils had an output whose phase and amplitude differed from the $\mathrm{X}$ and $\mathrm{Y}$ search coil outputs, depending upon the location and direction of such currents with respect to the moving. spacecraft.

\section{COMPARISON OF SIMULTANEOUS DATA}

In comparing search coil magnetometer data and precipitating particle data, the limitations of the two experiments greatly affect the success of correlation attempts. The search coil magnetometer responds to changes in $\vec{B}$ due to nearby currents without actually passing into or through a current containing region. The particle detectors respond on $1 \mathrm{y}$ to particles actually reaching the instruments, and then only if their energies lie within definite energy ranges.

An example of simultaneous data from the two experiments is presented in Figure 2, which shows about four seconds of data acquired near 21 hours MLT at $\Lambda \approx 73.3^{\circ}$. The top portion of the figure shows the near $0^{\circ}$ pitch angle particle fluxes at $0.7 \mathrm{keV}, 2.3 \mathrm{keV}$, and $7.3 \mathrm{keV}$, and the ratio of the $0^{\circ}$ flux to the $60^{\circ}$ flux at the energy of $2.3 \mathrm{keV}$; the $\mathrm{X}, \mathrm{Y}$, and $\mathrm{Z}$ search coil magnetometer responses are shown in the bottom of the figure. Barrow, Alaska was almost directly under the satellite at the time of the pass, and its magnetometer traces displayed a magnetic bay of $\sim 300 \gamma$ in the $\mathrm{H}$ component and $\mathrm{a} \sim 200 \gamma$ decrease in the $\mathrm{Z}$ component at that time. 
It is first noticed that in general the search coil outputs had their largest amplitudes and variations where the particle fluxes were high. Several current configurations can be tentatively identified by comparing the gross features of the search coil waveforms with the mode 1 responses shown in Figure 1 . In region $A$, defined by the 0.2 second burst of $2.3 \mathrm{keV}$ electrons with an anisotropy factor of about 10 , the $X$ and $Y$ search coils produced a waveform not unlike that resulting from passage through a circular current region. More clearly identifiable is the current system B of circular cross-section, again characterized by $2.3 \mathrm{keV}$ electrons with a very high degree of anisotropy. In fact we will show below that for this case there is excellent agreement between the magnetometer fluctuations calculated from the measured currents and that measured by all three search coils. The high latitude cut-off of $7.3 \mathrm{keV}$ electron precipitation at $\mathrm{C}$ can be identified as a temporal variation in the current through the $\mathrm{X}$ and $\mathrm{Y}$ waveforms.

Thus, despite the large quantity of OGO-4 data acquired, only a limited number of successful one-to-one particle and field correlations were expected, since the chief problem was finding isolated, clearly definable current configurations.

We will next consider in detail several examples where successful correlations could be made. From the current densities measured in the bandpasses of the particle detectors, and with certain assumptions on the nature of the electron precipitation, search coil magnetometer responses car ld be computed and compared with the observed search coil outputs . 
The current density is calculated from the particle measurements by

$$
J=\iint e \frac{d^{2} F}{d \alpha d E} \cos \alpha(2 \pi \sin \alpha) d \alpha d E
$$

where

e is the electronic charge

$\frac{\mathrm{d}^{2} \mathrm{~F}}{\mathrm{~d} \alpha \mathrm{E}}$ is the flux at the energy $\mathrm{E}$ and pitch angle $\alpha$

and $\cos \alpha$ is required because only the parallel component of the velocity contributes the current.

The integration over pitch angle was performed at each of the three energies $0.7,2.3$ and $7.3 \mathrm{keV}$ (negligible fluxes were encountered at $23.8 \mathrm{keV})$. At the two lower energies, the four point pitch angle distribution measured at $2.3 \mathrm{keV}$ was used, with linear interpolations between the fluxes at the angles measured. The flux was always assumed to be isotropic at $7.3 \mathrm{keV}$. This first set of integrations yielded a three point current density spectrum (amps/m²-keV). The second integral over energy was then performed using power law spectra between the three points.

As a first example, we have taken the spatial current variation encountered in region B of Pass 2988 (Figure 2). The current density J as a function of time is shown at the top of Figure 3. A smooth curve, indicated by the dashed line, was then fit to the measured current density. Using this analytic representation for J, Ampere's law was used to calculate the magnetic field produced by this current distribution:

$$
\oint \overrightarrow{\mathrm{B}} \cdot \overrightarrow{\mathrm{dl}}=\mu_{\mathrm{o}} \int_{\mathrm{A}} \overrightarrow{\mathrm{J}} \cdot \hat{\mathrm{n}} \mathrm{dA},
$$

where $A$ is the area inside the closed path dl. 
We then have

$$
B=\frac{\mu_{0}}{R} \int_{0}^{R} J(r) r d r,
$$

where we have assumed that the current form was cylindrical in shape and the satellite effectively traversed the center.

The resultant model magnetic field is shown in the center of the figure. Since the current variation was assumed to vary spatially, equation 2 then takes the form

$$
V=-A \vec{n} \cdot(\vec{v} \cdot \vec{\nabla}) \vec{B}
$$

Thus, by taking the spatial derivative of the computed model magnetic field, and taking into account the effective search coil area, spacecraft velocity, search coil unit vectors and dot products, we can predict the search coil response to the current form. Unit vectors for the $X, Y$, and $Z$ search coils are

$$
\begin{aligned}
& \vec{n}_{X}=(-1 / \sqrt{2}, 0,-1 / \sqrt{2}) \\
& \vec{n}_{Y}=(-1 / \sqrt{2}, 0,1 / \sqrt{2}) \\
& \vec{n}_{Z}=(0,1,0)
\end{aligned}
$$

From equations 8 and 9, we obtain the induced voltages in the three axes of the magnetometer due to a spatially varying current form to be

$$
\begin{aligned}
& \mathrm{V}_{\mathrm{X}}=1 / \sqrt{2} \mathrm{~A} \mathrm{v} \frac{\partial}{\partial \mathrm{y}} \mathrm{B}_{\mathrm{x}} \\
& \mathrm{V}_{\mathrm{Y}}=1 / \sqrt{2} \mathrm{~A} \mathrm{v} \frac{\partial}{\partial \mathrm{y}} \mathrm{B}_{\mathrm{X}} \\
& \mathrm{V}_{\mathrm{Z}}=-\mathrm{A} \mathrm{v} \frac{\partial}{\partial \mathrm{y}} \mathrm{B}_{\mathrm{y}}
\end{aligned}
$$


for this case where the spacecraft velocity vector was in the $-y$ direction. The predicted $X$ axis search coil response, computed using the curve fit to the measured current density and equation 10 , is shown by the dashed line in the lower portion of Figure 3 . Excellent agreement is seen between the predicted and measured $X$ axis search coil output. Note in equation 10 and in Figure 2 that the calculated and measured $\mathrm{X}$ and $\mathrm{Y}$ axis wave forms should be and are identical. We can utilize the phase relationships between the $\mathrm{X}, \mathrm{Y}$, and $\mathrm{Z}$ axis search coil traces to determine the actual location of the center of the current region with respect to the satellite. We have indicated the signs of the magnetic field components $B_{x}$ and $B_{y}$ in Figure 4, where we are looking down at the current. It is evident that different search coil outputs will result depending on which side of the "center" of the current the satellite passes (the satellite velocity direction is known to be in the $-y$ direction for this first example). The key waveform for this determination is from the $\mathrm{Z}$ axis, where it is noted in Figure 2 that there is first a large positive signal followed by a symmetric negative signal in region $B$. Noting that the $Z$ axis signal depends upon the negative gradient of $\mathrm{B}_{\mathrm{y}}$, such a waveform will be obtained if the satellite passed through quadrants 1 and then 4 . On the other hand, the opposite polarities would have been obtained had it passed through quadrants 2 and then 3 .

Therefore, we conclude from these calculations that the majority of the current distribution was actually measured by the particle 
detectors since the amplitudes of the calculated and measured signals agree, the current configuration was basically cylindrical in form, and that the satellite passed somewhat to the side of the center of the current region.

As a second example of a comparison between predicted and measured search coil responses, we consider one second of data from Pass 3179. In the top portion of Figure 5 we have plotted the current density measured by the particle detectors integrated over pitch angle and energy (from 0.7 to $7.3 \mathrm{keV}$ ), to which a smooth curve (the dashed line) was fit, as in the previous example. This current form was also assumed to be spatially varying, and by the same method used in the previous example, a model magnetic field was computed, which is indicated in the center of Figure 5. At the bottom of the figure we have plotted the $Y$ axis search coil signal during this time period (solid curve).

Following the procedure used in the previous example, and again using equation 10 , we computed a predicted output signal for the $Y$ axis search coil, which is plotted as a dashed curve at the bottom of Figure 5. Agreement between the two curves is seen to be excellent. As in the previous example, the spacecraft velocity was in the $-y$ direction, and we can again employ the phase relationship between the $X, Y$, and $Z$ axis search coil outputs to determine the orientation of the current with respect to the satellite. The $Z$ axis signal is first negative and then positive (see Figure 7), the opposite of the previous example, so we can conclude that the spacecraft traversed quadrants 2 and then 3 of Figure 4. 
In the next example we shall consider a portion of data from Pass 3223 (Figure 6), in which the current variation was assumed to be temporal; i.e., the particle detectors and magnetometer were exposed to the field-aligned current as it increased and then decreased, without undergoing any relative spacecraft-current motion. For the case of a temporal current variation, equation 2 takes the form

$$
V=-A \vec{n} \cdot \frac{\partial \vec{B}}{\partial t}
$$

and inserting the unit vectors from equation 9, we find that the induced voltages in each search coil are given by

$$
\begin{aligned}
& \mathrm{V}_{\mathrm{X}}=1 / \sqrt{2} \mathrm{~A} \frac{\partial \mathrm{B}_{\mathrm{x}}}{\partial t} \\
& \mathrm{~V}_{\mathrm{Y}}=1 / \sqrt{2} \mathrm{~A} \frac{\partial \mathrm{B}_{\mathrm{X}}}{\partial \mathrm{t}} \\
& \mathrm{V}_{\mathrm{Z}}=-\mathrm{A} \frac{\partial \mathrm{B}_{\mathrm{y}}}{\partial \mathrm{t}}
\end{aligned}
$$

Using the information in Figure 4 with the equations, it can be shown that the waveforms from all three magnetometers can be exactly in phase only for the temporal case (if the satellite is in quadrants 1 or 3 of Figure 4), but never in the cylindrical current configuration. We note in Figure 8 (bottom) that indeed the $V_{X}$ and $V_{Z}$ waveforms have this property.

The current density shown by the solid curve in the top portion of Figure 6 was calculated by integrating the fluxes only from 0.7 to 2.3 $\mathrm{keV}$, since fluxes of 7.3 and $23 \mathrm{keV}$ electrons were essentially zero 
during this time. A smooth curve was then fit to this current density profile, as shown by the dashed line. Equations 12 show that the search coil output in this case is simply related to the time derivative of the magnetic field. The time derivative of $B$ was calculated from the $t$ ime derivative of $\mathrm{J}$ using the smooth form of $\mathrm{J}$. In doing this we have assumed that the current region was cylindrical with a spatially uniform current density out to the distance of the satellite. Therefore, with $J$ only a function of time, equation 7 can be written

$$
\frac{\partial B}{\partial t}=\frac{\mu_{0}}{R} \frac{\partial}{\partial t}\left[\int_{0}^{R} J(t) r d r\right]=\frac{\mu_{0}}{2} R \frac{\partial J}{\partial t}
$$

Applying equations 12 to this derivative we calculated the $\mathrm{Z}$ axis search coil response to the time varying current. A comparison of the results plotted in the bottom of Figure 6 with the measured waveform from the $Z$ axis coil shows good agreement in shape. A variable of the calculation was the radius $\mathrm{R}$, which was selected to be $3 \mathrm{~km}$ to give agreement in amplitude between the measured and calculated curves. The distance traveled by the satellite during the event must be small compared to $2 \mathrm{R}$ for the assumption of a temporal event to be valid. Since the event was $0.3 \mathrm{sec}$ in duration, during which the satellite moved slightly over $2 \mathrm{~km}$, our assumption is reasonably valid.

The several examples presented above do show that in some cases we can unambiguously identify the nature of a current form and the particle fluxes constituting it. In Figure 7 we present three examples of simultaneous data indicating the presence of spatially varying current configurations. We have plotted differential fluxes of 0.7 and $2.3 \mathrm{keV}$ electrons 
at the top for each example, as well as the ratio of $2.3 \mathrm{keV}$ fluxes with $0^{\circ}$ pitch angle to those with $60^{\circ}$ pitch angle. The simultaneous $\mathrm{X}, \mathrm{Y}$, and $Z$ axis search coil responses are plotted beneath the particle fluxes. One of these examples (Pass 3179 ) has already been considered in detail. Note in all three cases the $X$ and $Y$ waveforms are in phase, but out of phase with $\mathrm{Z}$.

Three examples of simultaneous data which we have identified as signalling time varying currents are presented in Figure 8 . Again one of the examples was previously considered in detail. Note in all three cases the three waveforms were all in phase. Note also that the magnetometer signatures in these three examples, as well as those in Figure 7 , were well isolated from other current signatures. Only the portion of Pass 2590 shown in Figure 8 was at a time of relatively low and isotropic $2.3 \mathrm{keV}$ flux (although the $0.7 \mathrm{keV}$ flux was high). In the other examples presented in this and the previous figure, the fluxes at both 0.7 and $2.3 \mathrm{keV}$ were relatively high and quite field-aligned at $2.3 \mathrm{keV}$ (ratio of $0^{\circ}$ pitch angle flux to $60^{\circ}$ flux $\geq 2$ ).

We conclude from a detailed analysis of the pe particular cases that the 0G0-4 particle detectors measured a significant portion of the fieldaligned currents on the night side. In addition, it appears that the field-aligned currents usually occurred in the regions of field-aligned particle fluxes. We can use, then, the regions of field-aligned particle measurements as an indicator of the field-aligned current regions, at least on the night side. 
REGIONS OF OCCURRENCE

It is of interest then to compare statistically the high-latitude regions where field-aligned electron precipitation is observed with the regions where magnetic fluctuations, indicative of the presence of fieldaligned currents, are often found. Figure 9 contains a superposition of regions where high fluxes of field-aligned $2.3 \mathrm{keV}$ precipitation were observed (from Berko, 1973), regions where the oGo-4 search coil magnetometers recorded fluctuations, and a "maximum" region where $\underline{\text { zmuda et al. }}$. (1970) observed transverse magnetic disturbances greater than $\sim 30 \gamma$. Coincidence between the regions of field-aligned particle precipitation and magnetic fluctuations, both from $0 G 0-4$ data and the data of Zmuda et al., is quite good, except between 8 and 14 hours MLT, where very few $2.3 \mathrm{keV}$ field-aligned precipitation events were detected. However, particle precipitation at these local times has been shown to consist primarily of electrons with energies in the hundreds of electron volts (Hoffman and Berko, 1971b; Heikkila and Winningham, 1971). A11 three regions shown in Figure 9 share the feature that the lower boundary is at higher latitudes during the dayside hours than in the near-midnight hours.

Hoffman (1972) has compared the quiet time (Kp less than or equal to $2+$ ) regions of magnetic disturbance of Zmuda et a1. with the regions of soft ( $0.7 \mathrm{keV})$ electron precipitation, when $\mathrm{Kp}$ was less than or equal to 2 (from Hoffman and Berko, 1971a), and found excellent congruity. In fact, since electrons with energies of $0.7 \mathrm{keV}$ and below are the primary electron influx to the dayside auroral regions (Hoffman and Berko, 1971a), 
they are the principal electrons on the dayside which are, undoubtedly, associated with the transverse magnetic disturbances seen there so frequent $1 y$ by the 0G0-4 search coil magnetometers and by Zmuda et a1. (1970).

\section{FIELD-ALIGNED CURRENT MAGNITUDES}

The current densities calculated from the measurements by the Auroral Particles Experiment range up to about $5 \times 10^{-5}$ amps $/ \mathrm{m}^{2}$ in the examples discussed above. These values fall well within the range of measured, inferred and theoretical values of field-aligned currents which have appeared in the 1iterature and are 1isted in Table 1 . While the counting rates from the particle detectors in the examples were usually quite high, they were by no means the maximums ever observed in passes through the midnight region during substorms. To estimate the total current out of the ionosphere due to electron influxes, we first find the current in a cylindrical current region. Using a radius of $\sim 1 \mathrm{~km}$ (the distance the satellite moved in $0.1 \mathrm{sec}$ ) and a current density of $5 \times 10^{-5} \mathrm{amps} / \mathrm{m}^{2}$, there results a current of 150 amps per cylinder. Such current regions might be encountered 6 times per pass (See e.g. Figure 2) in a width of 3 seconds, or $24 \mathrm{~km}$, for a density of one every 4 kilometers. Assuming a longitudinal extent of $1,000 \mathrm{~km}$ at this density ( 2 hours local time at $70^{\circ}$ latitude), there results about 1,500 current regions. Therefore, the total current out of the ionosphere is about $2 \times 10^{5}$ amps, again in good agreement with the estimates listed in Table 1. 


\section{SUMMARY}

Data from the OGO-4 Auroral Particles Experiment and Search Coil Magnetometer Experiment have been compared at times when simultaneous, high time resolution measurements were available at high latitudes. Magnetometer fluctuations indicative of field-aligned current were frequently seen at all local times. Definite correlations between lowenergy particle fluxes and field-aligned current signatures were found during many satellite passes. Using several simple idealized models of current configurations, both spatial and temporal current variations were distinguishable, although such a definite categorization was not always possible.

Comparisons of measured magnetic field disturbances with those calculated from measured current densities, using the simple models of current configurations, give excellent agreement in certain late evening hour cases. Field-aligned currents in these hours were detected in the region of high field-aligned particle flux occurrence. Most of the current in this region was carried by particles with energies greater than $0.7 \mathrm{keV}$. It is probable that dayside field-aligned currents consist primarily of electrons with energies less than $\sim 1 \mathrm{keV}$, which are the prime charge carriers in these hours.

\section{ACKNOWLEDGMENTS}

This report represents one aspect of research done by the Jet Propulsion Laboratory for NASA under NASA Contract NGR-05-007-276. Financial support for the work at the University of California was provided by JPL under Contract 950403 . 


\section{1 \\ REFERE NCES}

Ackerson, K. L., and L. A. Frank, Correlated satellite measurements of low-energy electron precipitation and ground-based observations of a visible auroral arc, J. Geophys. Res., 77, 1128-1136, 1972.

Akasofu, S.-I., and C.-I. Meng, A study of polar magnetic substorms, J. Geophys. Res., 74, 293-313, 1969.

Armstrong, J. C., and A. J. Zmuda, Field-aligned current at $1100 \mathrm{~km}$ in the auroral region measured by satellite, J. Geophys. Res., 도, 71227127,1970 .

Berko, F. W., Distributions and characteristics of high-latitude fieldaligned electron precipitation, J. Geophys. Res., 78, 1973, in press. Boström, R., Currents in the ionosphere and magnetosphere, Ann. Geophys., $\underline{24}, 681-694,1968$.

Chase, L. M., Energy spectra of auroral zone particles, J. Geophys. Res., $\underline{75}, 7128-7139,1970$.

Choy, L. W., R. L. Arnoldy, W. Potter, P. Kintner, and L. J. Cahil1, Jr., Field-aligned particle currents near an auroral arc, J. Geophys. Res., 76 $, 8279-8298,1971$.

Cloutier, P. A., H. R. Anderson, R. J. Park, R. R. Vondrak, R. J. Spiger, and B. R. Sande1, Detection of geomagnetically aligned currents associated with an auroral arc, J. Geophys. Res., 75, 2595-2600, 1970. Cummings, W. D., and A. J. Dessler, Field aligned currents in the magnetosphere, J. Geophys. Res., 72, 1007-1013, 1967.

Evans, D. S., Low energy charged-particle detection using the continuouschannel electron multiplier, Rev. Sci. Instruments, 36, 375-382, 1965. 


\section{8}

- 2 -

Evans, D., B. Maehlum, and T. Wedde, High latitude observations of field aligned electron beams, (abstract), EOS Trans. AGU, 53, 731, 1972.

Frandsen, A. M. A., R. E. Holzer, and E. J. Smith, OGO search coil magnetometer experiments, IEEE Trans. Geoscience Electronics, GE-7, 61-74, 1969.

Frank, L. A., Plasma in the earth's polar magnetosphere, J. Geophys. Res., 76, 5202-5219, 1971 .

Haerende1, G., P. C. Hedgecock, and S.-I. Akasofu, Evidence for magnetic field aligned currents during the substorms of March 18, 1969, J.Geophys. Res., 76, 2382-2395, 1971 .

Heikkila, W. J., and J. D. Winningham, Penetration of magnetosheath plasma to low altitudes through the dayside magnetospheric cusps, J. Geophys. $\underline{\text { Res., }}$ 76, 883-891, 1971 .

Hoffman, R. A., Properties of low energy particle impacts in the polar domain in the dawn and dayside hours, in Magnetosphere-Ionosphere Interactions, pp. 117-138, ed. K. Folkestad, Universitetsforleget, Oslo, 1972.

Hoffman, R. A., and F.W. Berko, Primary electron influx to dayside auroral ova1, J. Geophys. Res., 76, 2967-2976, 1971a.

Hoffman, R. A., and F. W. Berko, on the dual nature of the auroral oval, (abstract), EOS, Trans. AGU, 52, 325, 1971b. Hoffman, R. A., and D. S. Evans, Field-aligned electron bursts at high latitudes observed by OG0 4, J. Geophys. Res., 73, 6201-6214, 1968. 
O'Brien, B. J., and D. L. Reasoner, Measurements of highly collimated short-duration bursts of auroral electrons and comparison with existing auroral mode1s, J. Geophys. Res., 26, 3258-8278, 1971 .

Park, R. J., and P.A. Cloutier, Rocket-based magnetometer measurement of birkeland currents related to an auroral arc and electrojet, $\mathrm{J}$. Geophys. Res., 76, 7714-7733, 1971 .

Vondrak, R. R., H. R. Anderson, and R. J. Spiger, Rocket-based measurements of particle fluxés and currents in an auroral arc, J. Geophys. Res., 76 , $7701-7713,1971$.

Whalen, B. A., and I. B. McDiarmid, Observations of magnetic-field-a1igned aurora1-electron precipitation, J. Geophys. Res., 77, 191-202, 1972 .

Zmuda, A. J., J. H. Martin, and F. T. Heuring, Transverse magnetic disturbances at 1100 kilometers in the auroral region, J. Geophys. Res., 71, 5033-5054, 1966 .

Zmuda, A. J., F. T. Heuring, and J。 H. Martin, Dayside magnetic disturbances at $1100 \mathrm{~km}$ in the auroral oval, J. Geophys. Res., 72, 1115-1117, 1967. Zmuda, A. J., J. C. Armstrong, and F. T. Heuring, Characteristics of transverse magnetic disturbances observed at 1100 kilometers in the auroral oval, J. Geophys. Res., 75, 4757-4762, 1970. 


\section{FIGURE CAPTIONS}

Figure 1. Three types of simple field-aligned current variations, their resultant magnetic fields, and the expected $\dot{B}$ for: top - purely temporal variations; center - cylindrical, spatial variation; bottom - spatial variation, sheet current.

Figure 2. Simultaneous electron fluxes and search coil outputs during an evening hour pass. Magnetic signatures of several identifiable current forms are shown between the $X$ and $Y$ search coil traces.

Figure 3. Top-calculated current density (solid line) and smooth curve representation (dashed 1ine) for the spatial current variation in region $B$ of Figure 2; center - computed magnetic field; bottom - actual $\mathrm{X}$ axis search coil response (solid curve) and predicted $X$ axis search coil response (dashed curve).

Figure 4. Signs of the components of the magnetic field in the $x-y$ plane looking down on the (upward) field-aligned current.

Figure 5. Similar to Figure 3 for a portion of data from Pass 3179 , except that the actual and predicted $Y$ axis search coil outputs are shown. The data were taken at $\Lambda=70.5^{\circ}$ and 19.8 hours MLT.

Figure 6. Similar to'Figure 3 for the current variation during Pass 3223 identified as temporal, with actual $\mathrm{X}$ and $\mathrm{Z}$ axis search coil responses (solid lines) and predicted $Z$ axis response (dashed 1ine) shown in the bottom of the figure. These data were obtained at $\Lambda=71.0^{\circ}$ and 19.5 hours MLT.

Figure 7. Three examples of simultaneous electron fluxes and search coil outputs during simple, purely spatial current variations, as determined by comparison of the search coil output and $\dot{B}$ mode $1 \mathrm{~s}$ from Figure 1 . 
Figure 8. Similar to Figure 7 for three examples of temporal current variations .

Figure 9. Comparison of regions where field-aligned $2.3 \mathrm{keV}$ electron precipitation was observed by $0 \mathrm{GO}-4$ (Berko, 1973) with the regions where $0 G 0-4$ search coil magnetometer fluctuations were observed and where transverse magnetic distrubances were observed by Zmuda et a1. (1970). 


\section{FIELD-ALIGNED CURRENT VALUES}

\section{AUTHOR(S)}

Boström $(1968)^{3}$

Zmuda et al. $(1970)^{2}$

Choy et al. $(1971)^{1}$

Cloutier et al $(1970)^{1}$

Frank $(1971)^{1}$

Park and Cloutier $(1971)^{2}$

Vondrak et al (1971) 1

Whalen and McDiarmid $(1972)^{1}$

This Study (typica1)

* These were maximum values
$1=$ measured
$2=$ inferred
3 = theoretical

\section{CURRENT DENSITY $\left(\mathrm{amp} / \mathrm{m}^{2}\right)$}

$7.5 \times 10^{-6}$

$0.2-6.0 \times 10^{-6}$

$5 \times 10^{-6}-5 \times 10^{-7}$

$2 \times 10^{-5}$

$3 \times 10^{-7}$

$2 \times 10^{-5}$

$1.5 \times 10^{-5 *}$

$2 \times 10^{-4 *}$

$5 \times 10^{-5}$
CURRENT (amp)

Akasofu and Meng $(1969)^{3}$

$10^{6}$

Armstrong and Zmuda $(1970)^{2} 6.4 \times 10^{5} /$ sheet

Boström $(1968)^{3}$

$1.5 \times 10^{6} /$ sheet

Cummings and Dessler $(1967)^{2}$

$10^{5}$

Haerendel et a1. $(1971)^{2}$

$5 \times 10^{5}$

This Study 
TEMPORAL CURRENT VARIATION:

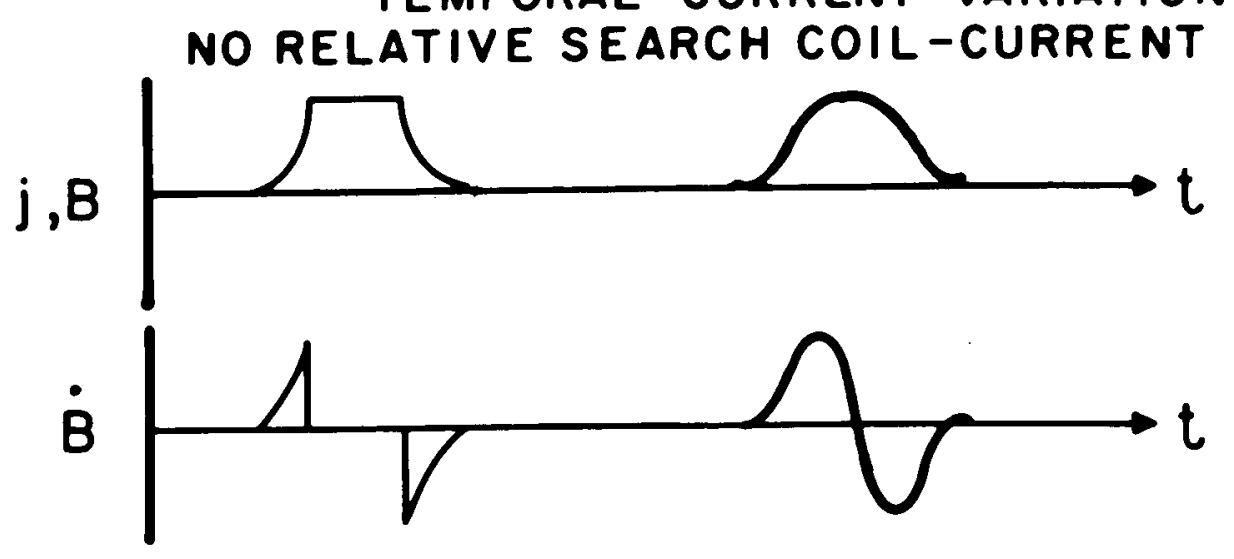

SPATIAL CURRENT VARIATION:

SEARCH COIL MOVING

THROUGH CYLINDRICAL CURRENT SYSTEM
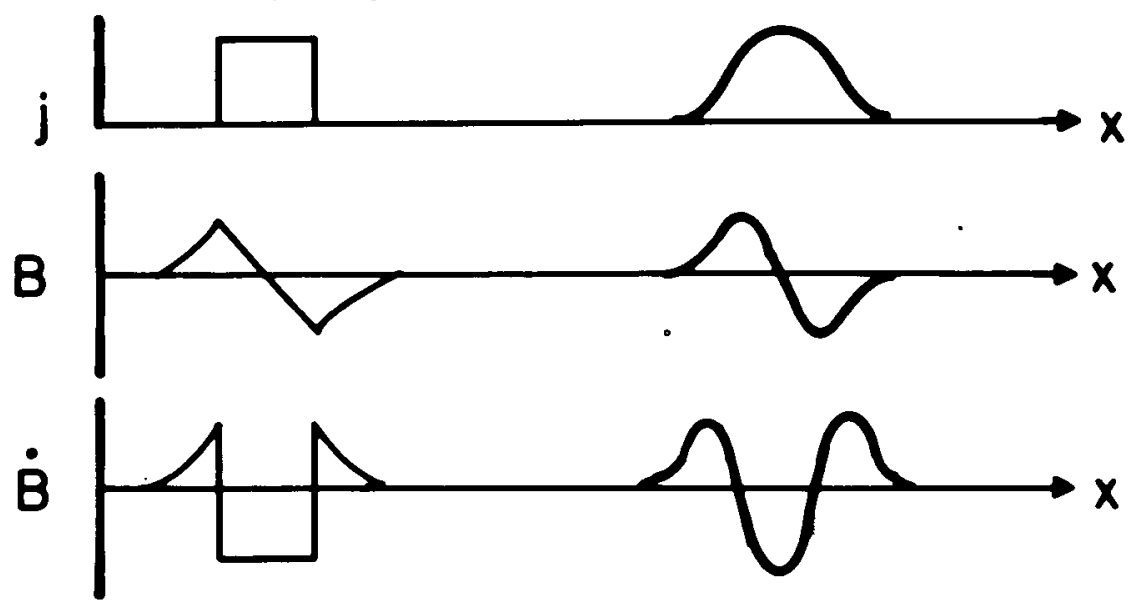

SPATIAL CURRENT VARIATION:

SEARCH COIL MOVING

THROUGH SHEET CURRENT
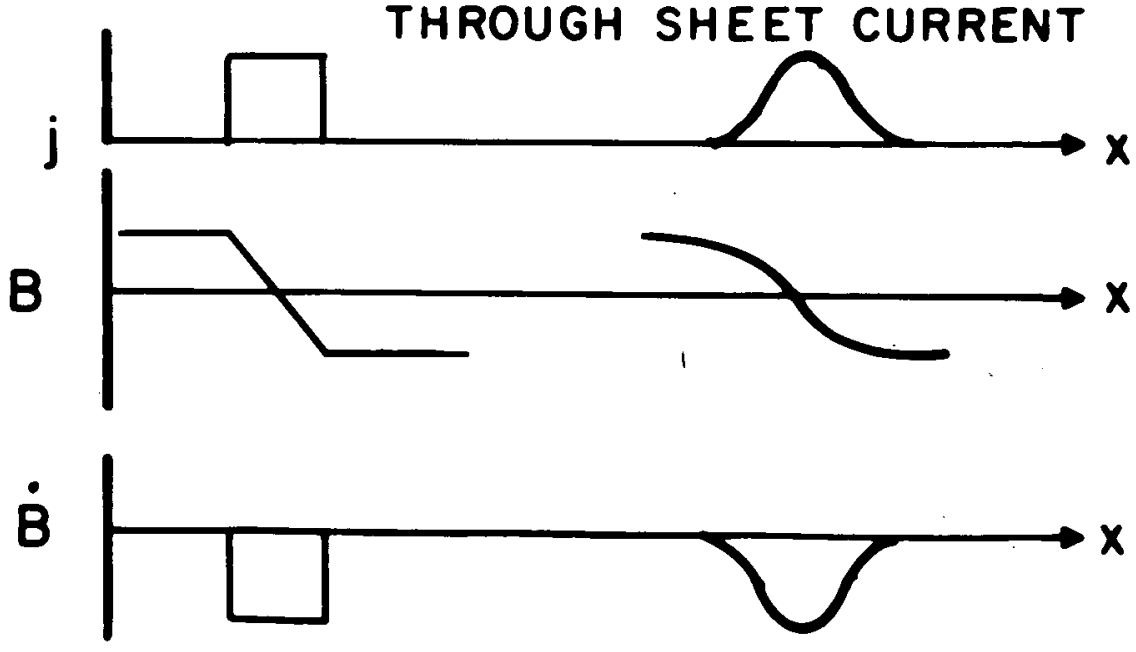

FIGURE 1 


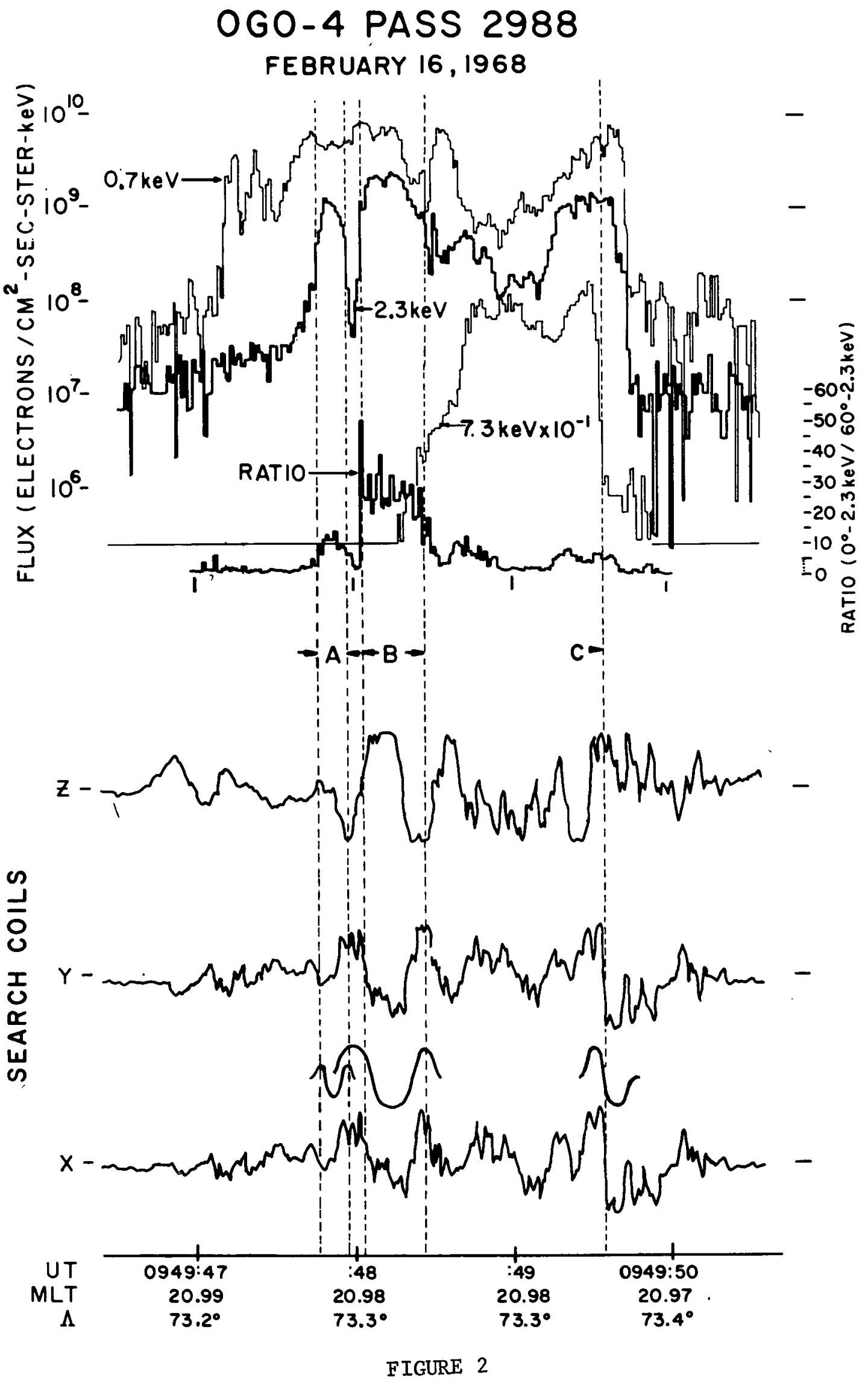




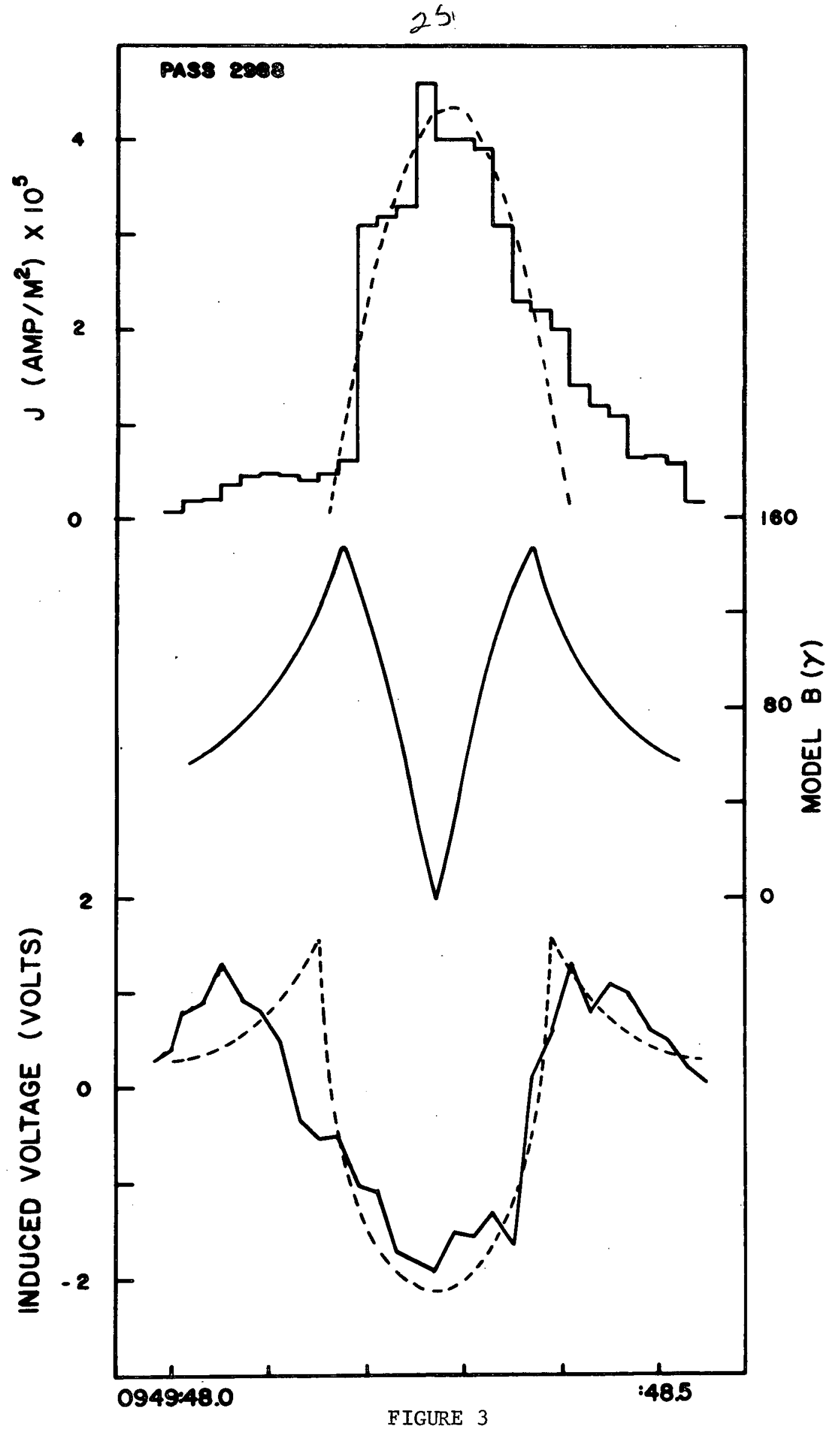


36

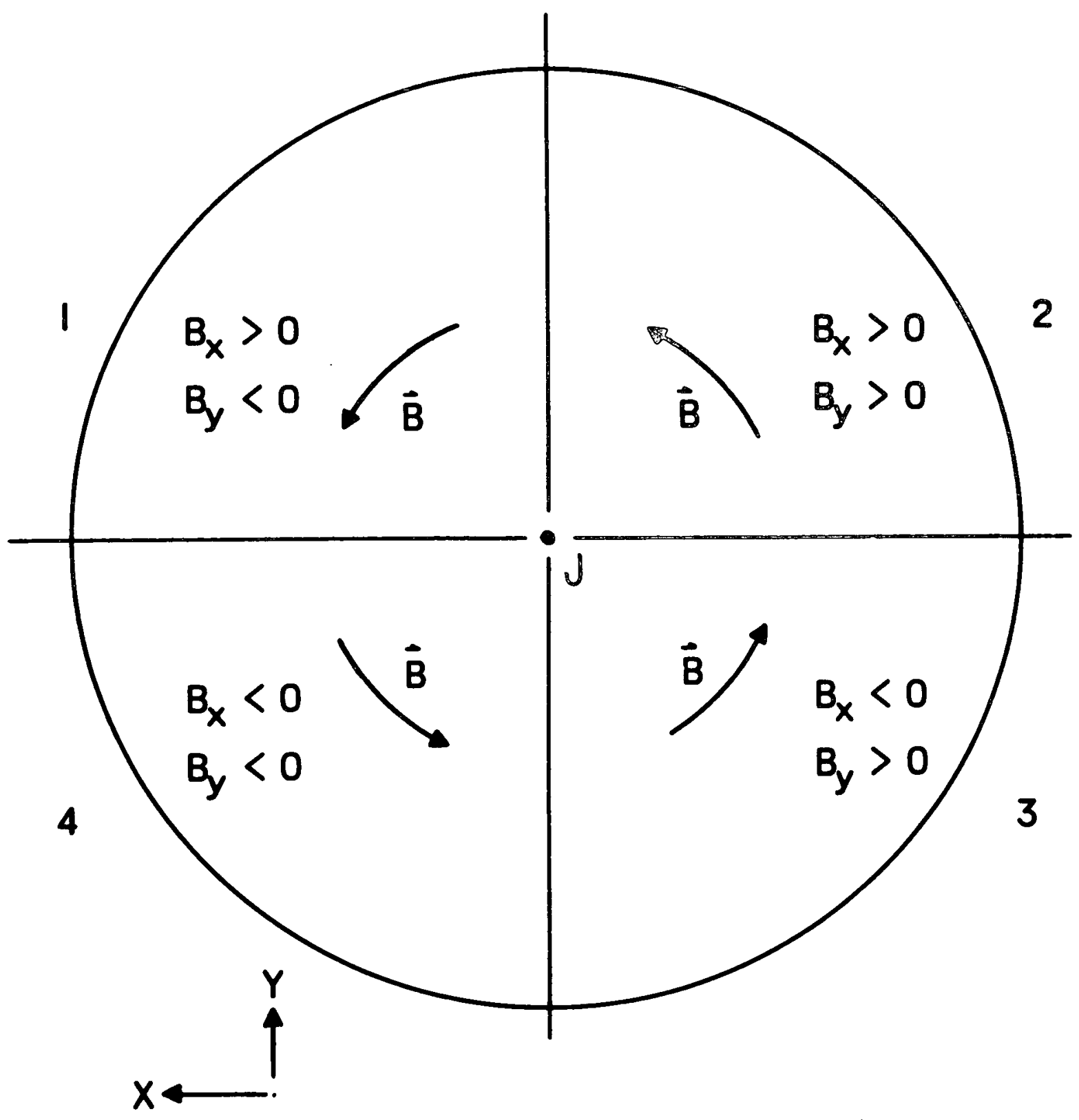

FIGURE 4 
27.

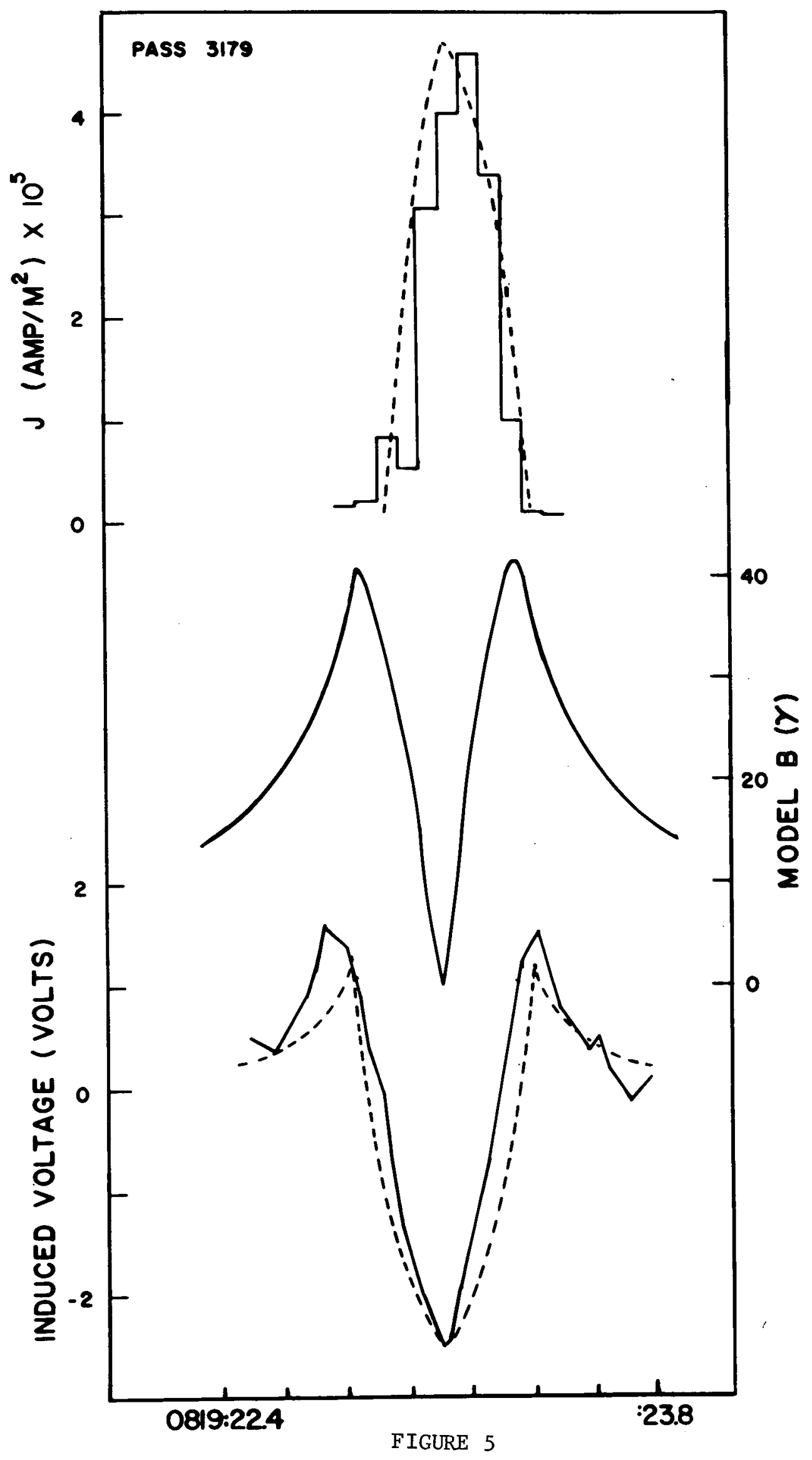


28

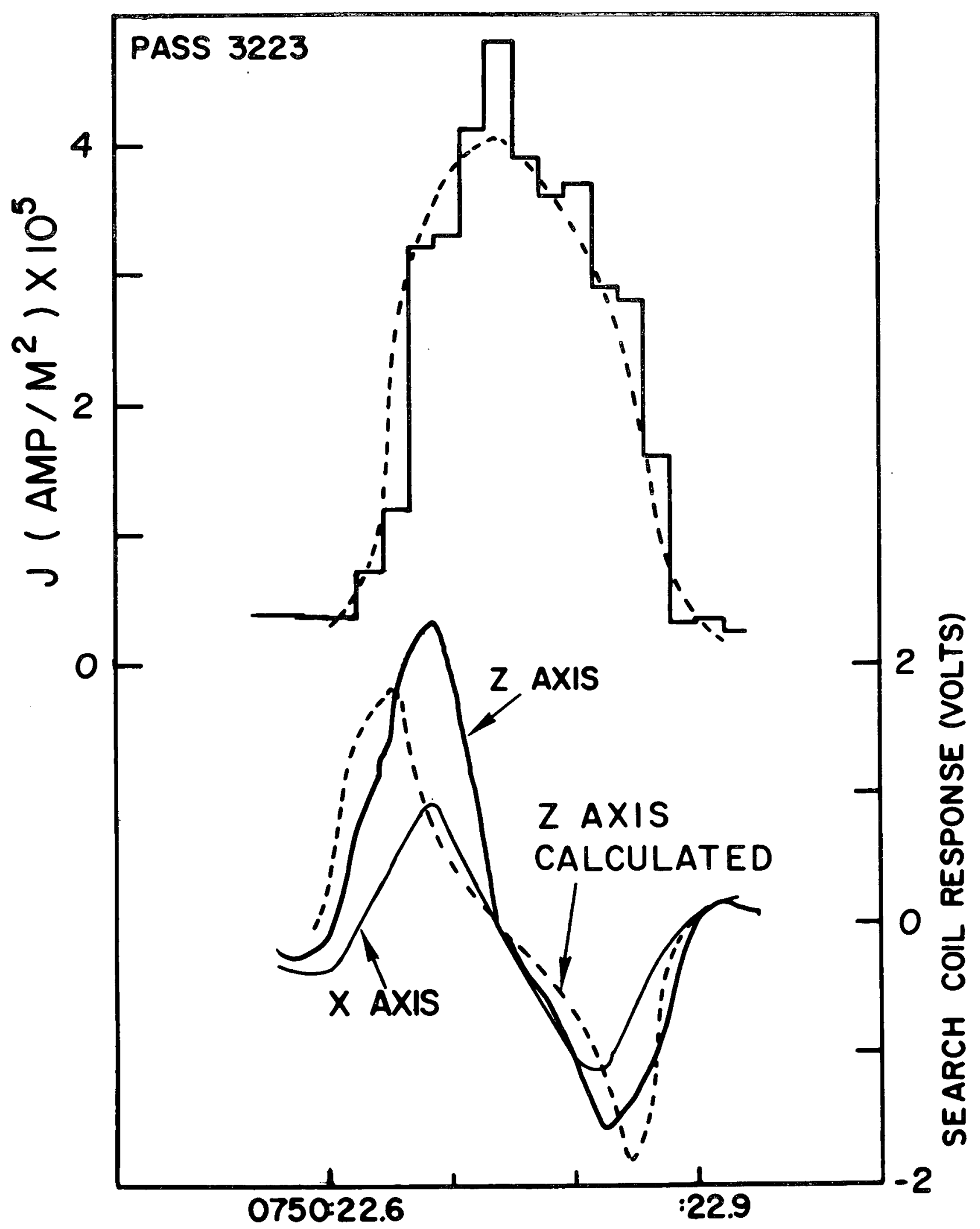

FIGURE 6 
29

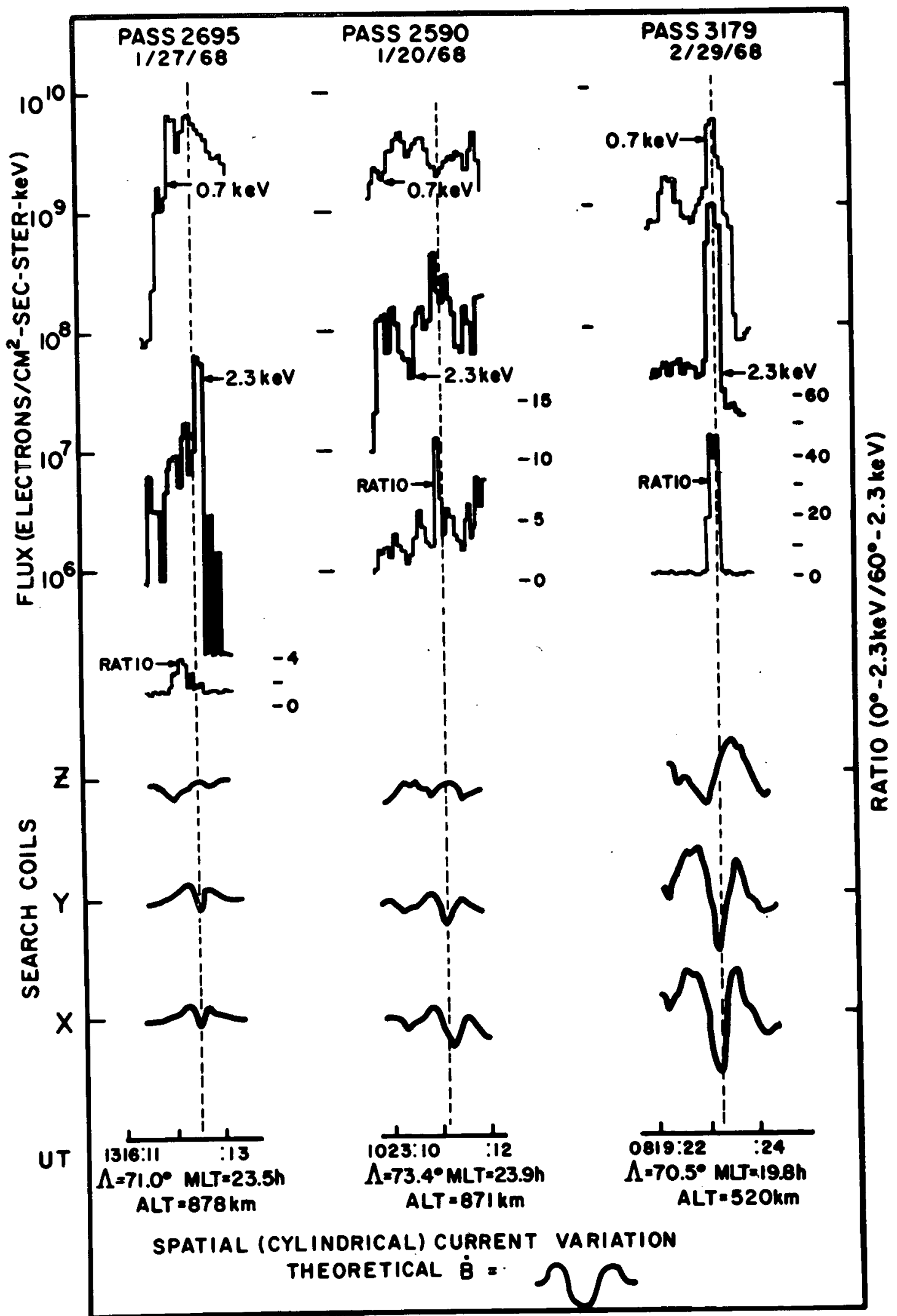




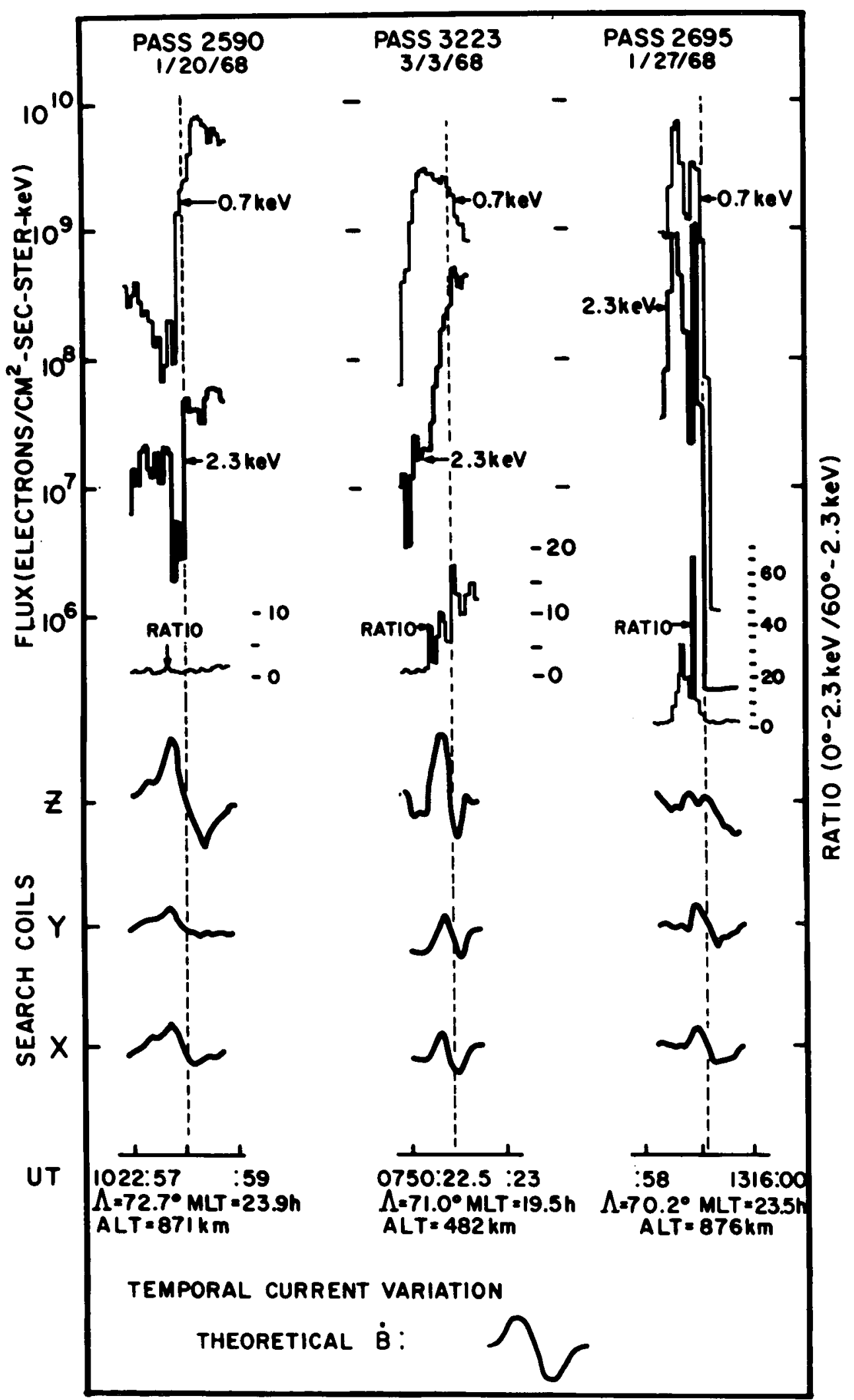




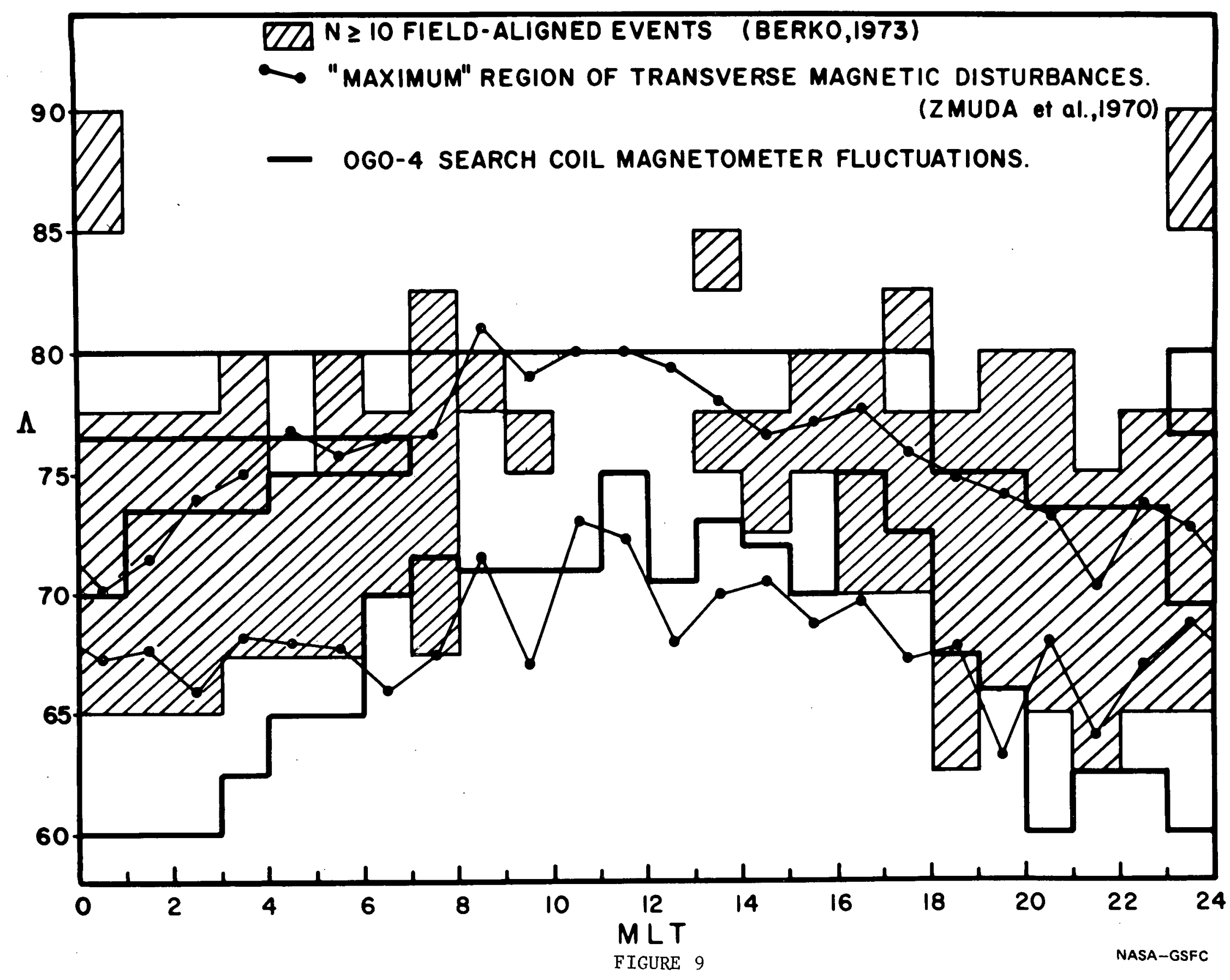

$M$ 